\title{
Strength exercise reduces hepatic pyruvate carboxylase and gluconeogenesis in DIO mice
}

\author{
Rodrigo Martins Pereira1,*, Kellen Cristina da Cruz Rodrigues ${ }^{1, *}$, Marcella Ramos Sant'Ana², \\ Guilherme Francisco Peruca1, Ana Paula Morelli3, Fernando M Simabuco3, Adelino S R da Silva ${ }^{4} 4,5$, \\ Dennys Esper Cintra², Eduardo Rochete Ropelle ${ }^{6}$, José Rodrigo Pauli6 and Leandro Pereira de Moura 1
}

1Exercise Cell Biology Lab, Faculty of Applied Sciences, State University of Campinas, Limeira, Brazil 2Laboratory of Nutritional Genomics, Faculty of Applied Sciences, State University of Campinas, Limeira, Brazil ${ }_{3}^{3}$ Multidisciplinary Laboratory of Food and Health, State University of Campinas, Faculty of Applied Sciences, Limeira, Brazil ${ }^{4}$ School of Physical Education and Sport of Ribeirão Preto, University of São Paulo (USP), Ribeirão Preto, São Paulo, Brazil

5Postgraduate Program in Rehabilitation and Functional Performance, Ribeirão Preto Medical School, USP, Ribeirão Preto, São Paulo, Brazil 6Laboratory of Molecular Biology of Exercise (LaBMEx), Faculty of Applied Sciences, State University of Campinas (UNICAMP), Limeira, São Paulo, Brazil

Correspondence should be addressed to L P de Moura: mouralp@unicamp.br

*(R M Pereira and K C C Rodrigues contributed equally to this work)

\begin{abstract}
Obesity is linked to a reduction in the control of hepatic glucose production, which is the primary mechanism related to fasting hyperglycemia and the development of type 2 diabetes mellitus (T2DM). The main system involved in hepatic gluconeogenesis synthesis is controlled by pyruvate carboxylase (PC), which increases in obesity conditions. Recently, we showed that short-term strength training is an important tool against obesity-induced hyperglycemia. As aerobic exercise can reduce the hepatic PC content of obese animals, we hypothesized that strength exercise can also decrease this gluconeogenic enzyme. Therefore, this study investigated whether the metabolic benefits promoted by short-term strength training are related to changes in hepatic PC content. Swiss mice were divided into three groups: lean control $(\mathrm{Ctl})$, obese sedentary (ObS), and obese short-term strength training (STST). The STST protocol was performed through one session/day for 15 days. The obese exercised animals had reduced hyperglycemia and insulin resistance. These results were related to better control of hepatic glucose production and hepatic insulin sensitivity. Our bioinformatics analysis showed that hepatic PC mRNA levels have positive correlations with glucose levels and adiposity, and negative correlations with locomotor activity and muscle mass. We also found that hepatic mRNA levels are related to lipogenic markers in the liver. Finally, we observed that the obese animals had an increased hepatic PC level; however, STST was efficient in reducing its amount. In conclusion, we provide insights into new biomolecular mechanisms by showing how STST is an efficient tool against obesityrelated hyperglycemia and T2DM, even without body weight changes.
\end{abstract}

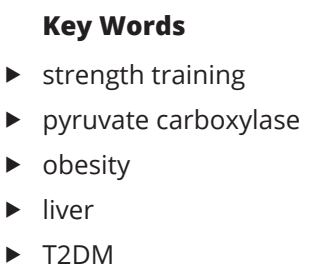


they showed normalized hepatic acetyl-CoA content, PC

\section{Introduction}

De novo synthesis of glucose from glycan precursors (nonsugars or non-carbohydrates), such as lactate, amino acids, and glycerol, is defined as gluconeogenesis. This synthesis is controlled by the liver and the cortex of the kidneys and is important for the maintenance of glycemic homeostasis, mostly under fasting conditions (Zhang et al. 2018). Due to insulin resistance, obese individuals present uncontrolled hepatic gluconeogenesis; thus, any derangement in this endogenous glucose production may contribute to the maintenance of fasting hyperglycemia and, consequently, to the development of type 2 diabetes mellitus (T2DM) (Magnusson et al. 1992, Gastaldelli et al. 2000, Basu et al. 2005). Therefore, it is of paramount importance to understand the mechanisms that underlie hepatic gluconeogenesis for the treatment and prevention of T2DM, providing new perspectives for glycemic control, since most of the current strategies are based on modulating insulin secretion and reducing insulin resistance.

Initially, there are mainly two mechanisms for hepatic gluconeogenesis to occur. First, inside the mitochondria, the main mechanism is controlled by the enzyme pyruvate carboxylase (PC), which forms oxaloacetate (OXA) from the carboxylation of pyruvate and provides substrate for the second step to start. In the cytosol, the gluconeogenic enzymes phosphoenolpyruvate carboxykinase (PEPCK) and glucose-6-phosphatase (G6Pase) continue the synthesis of a new glucose molecule from the OXA generated by the PC (Weber \& Cantero 1954, McGilvery \& Mokrasch 1956, Utter \& Keech 1960). In 2001, the enzymes PEPCK and G6Pase were listed as the main ones responsible for increasing hepatic gluconeogenesis (Yoon et al. 2001). However, in 2009, it was shown that fasting hyperglycemia was not associated with these enzymes in humans (Samuel et al. 2009), highlighting that more attention should be directed to the role of PC in the liver gluconeogenesis process (Jitrapakdee et al. 2008). This is because PC, by providing the substrate, is an indispensable enzyme for gluconeogenesis.

Gluconeogenesis, hepatic glucose production (HGP), and hepatic PC might be regulated by an indirect effect of insulin on suppressing white adipose tissue (WAT) lipolysis, which in turn decreases hepatic acetyl-CoA (an allosteric activator of PC), leading to reduced hepatic PC (Perry et al. 2015). On the other hand, high fat-fed rats showed insulin resistance in WAT, and increased PC activity; however, when these animals were treated with atglistatin, an inhibitor of adipose triglyceride lipase, activity, and HPG (Perry et al. 2015). An elegant study showed that diabetic animals have around 2.5 times more PC than control lean animals (Weinberg \& Utter 1980). In 2013, Kumashiro and collaborators showed that obese animals had increased hepatic PC levels and, after reducing its synthesis (using a specific antisense oligonucleotide ASO), it was possible to observe an improvement in insulin sensitivity, a reduction in endogenous glucose production, and, consequently, a reduction in hyperglycemia in obese rodents (Kumashiro et al. 2013). Furthermore, the authors showed that the relative hepatic PC levels were directly correlated with serum glucose and glycated hemoglobin levels in humans (Kumashiro et al. 2013), unlike the association of PEPCK and G6Pase with serum glucose (Samuel et al. 2009). Therefore, as PC has been highlighted as the main molecule involved in hepatic gluconeogenesis, studies aiming to reduce its activity are crucial in trying to control fasting hyperglycemia in diabetic subjects.

In this context, it is known that the regular practice of aerobic exercise is an important non-pharmacological tool that assists in the control of fasting hyperglycemia. Muñoz and colleagues showed that an 8-week aerobic training protocol decreased body weight, fasting hyperglycemia, and hyperinsulinemia in obese mice, as well as improving insulin signaling. Interestingly, these data were accompanied by a reduction in hepatic PC levels (Muñoz et al. 2018). On the other hand, the effects of strength physical exercise and body weight changes on PC levels were not addressed. Recently, we verified that a short-term strength exercise protocol reduced HGP, ameliorating glycemic homeostasis without body weight changes (Pereira et al. 2019). However, it was not described whether this improvement in glucose metabolism is linked to PC levels. Therefore, the present study evaluated the effects of short-term strength exercise on the hepatic PC levels and glucose homeostasis of diet-induced obese (DIO) mice, with no differences in body adiposity.

\section{Material and methods}

\section{Animals and diet}

All the animal procedures were previously approved by the Ethics Committee on Animal Use (CEUA) in Biological Sciences (UNICAMP - Campinas - SP, case number 4406-1) and carried out according to the Brazilian legislation on the scientific use of animals (Law No. 11,794, of October 8,2008$)$. In the present study, 8-week-old male 
The short-term strength training protocol started 48

Swiss mice were used, provided by the Multidisciplinary Center for Biological Research/UNICAMP. The animals arrived at 4 weeks old and were maintained in individual polyethylene cages with an enriched environment as previously described (Pereira et al. 2019). Briefly, animals were kept with a ratio of $12 \mathrm{~h}$ light: $12 \mathrm{~h}$ darkness, the temperature was controlled at $22 \pm 2^{\circ} \mathrm{C}$, and water and food (chow or high-fat diet) were offered ad libitum. More details about animal conditions were published before (Pereira et al. 2019).

The first step of this study was to induce obesity through diet, and at this point, the animals were distributed into two groups: control lean group (Ctl), fed a chow diet, and DIO group, fed a high-fat diet (HFD). The diet-induced obesity protocol lasted 14 weeks, and for the second step of this study, the obese group was equally redistributed according to the mice's body weight and fasting glycemia into two groups: (1) obese sedentary (ObS), consisting of obese mice that remained sedentary throughout the experiment, and (2) shortterm strength training (STST), consisting of mice that performed the strength training protocol. The HFD was prepared according to the American Institute of Nutrition (AIN-93G) guidelines (Reeves et al. 1993), and it was modified to contain $35 \%$ fat ( $4 \%$ soy oil and $31 \%$ lard) (Oliveira et al. 2015).

\section{Short-term strength training protocol}

The short-term strength training protocol was already published in detail by our research group (Pereira et al. 2019). Briefly, the protocol was performed on a ladder where the mice carried the load apparatus fixed with adhesive tape to their tails. Before the strength training protocol, the animals were adapted to the ladder and load apparatus for 5 consecutive days.

After the adaptation protocol, the mice rested for $48 \mathrm{~h}$. Then, the rodents were subjected to the maximal voluntary carrying capacity (MVCC) test to determine the maximum load with which each animal could climb the entire length of the ladder. The load for the first attempt at the MVCC test was equivalent to $75 \%$ of the animals' body weight. In the subsequent attempts, an incremental overload (5 g) was added in each further attempt to climb until the animal could no longer complete the entire course. The mice rested for $5 \mathrm{~min}$ in an individual cage between each attempt, and the heaviest overload carried in the last successful attempt was considered the animal's MVCC, and this value was used to prescribe the individual loads in the experiment. $\mathrm{h}$ after the MVCC determination. The STST consisted of 20 climbing series with an overload of $70 \%$ of the MVCC and with a rest interval of 60-90 s between sets. The mice were exercised for 5 consecutive days a week, followed by 2 days of rest, until they completed 13 sessions of physical exercise. After that, the mice underwent the pyruvate tolerance test or insulin tolerance test, and after $24 \mathrm{~h}$, the animals performed two more training sessions, totaling 15 sessions, and then they were euthanized (Fig. 1).

\section{Insulin tolerance test (ITT) and intraperitoneal pyruvate tolerance test (ipPTT)}

Eight hours after the end of the 13th exercise session, some of the animals were subjected to the ITT and the rest were subjected to the ipPTT, after $8 \mathrm{~h}$ of fasting. For the ITT, the mice received an intraperitoneal injection of recombinant human insulin (Humulin R) from Eli Lilly at a concentration of $1.5 \mathrm{U} / \mathrm{kg}$ of body weight. Blood samples were collected from the tail at $0,5,10,15,20,25$, and 30 min to determine the glycemic levels using a glucometer (Accu-Chek; Roche Diagnostics). Time 0 was measured before the insulin injection. The rate constant for plasma glucose disappearance (kITT) was calculated using the formula $0.693 /$ biological half-life $\left(t_{1 / 2}\right)$. Plasma glucose $t_{1 / 2}$ was calculated from the slope of the least squares analysis of the serum glucose concentration during the linear decay phase (Bonora et al. 1989). For the ipPTT, the animals were subjected to an intraperitoneal injection (i.p.) of pyruvate ( $2 \mathrm{~g} / \mathrm{kg}$ of sodium pyruvate) (Azis Científica ${ }^{\circledR}$, Cotia, SP, Brazil). The blood samples from the animals were drawn from the tail before the pyruvate injection, which was considered as time 0 , and 30, 60, 90, and 120 min after the injection to determine the blood glucose concentration. The ITT and ipPTT results were evaluated based on the areas under the serum glucose curves (AUC) during the test using the trapezoidal method (Matthews et al. 1990) in Microsoft Excel (2013) (Microsoft Corporation).

\section{Tissue extraction and immunoblotting analysis}

The euthanasia was performed $8 \mathrm{~h}$ after the 15 th exercise session, and during those $8 \mathrm{~h}$ the animals were also fasted. Ten minutes before the euthanasia, the animals received either human insulin $(8 \mathrm{U} / \mathrm{kg}$ body weight Humulin-R; Lilly) or saline via i.p. The animals were anesthetized via i.p. by the injection of chloral hydrate of ketamine (100 mg/kg, Parke-Davis, Ann Arbor, MI, USA) and xylazine (10 mg/kg, Rompun, Bayer), 

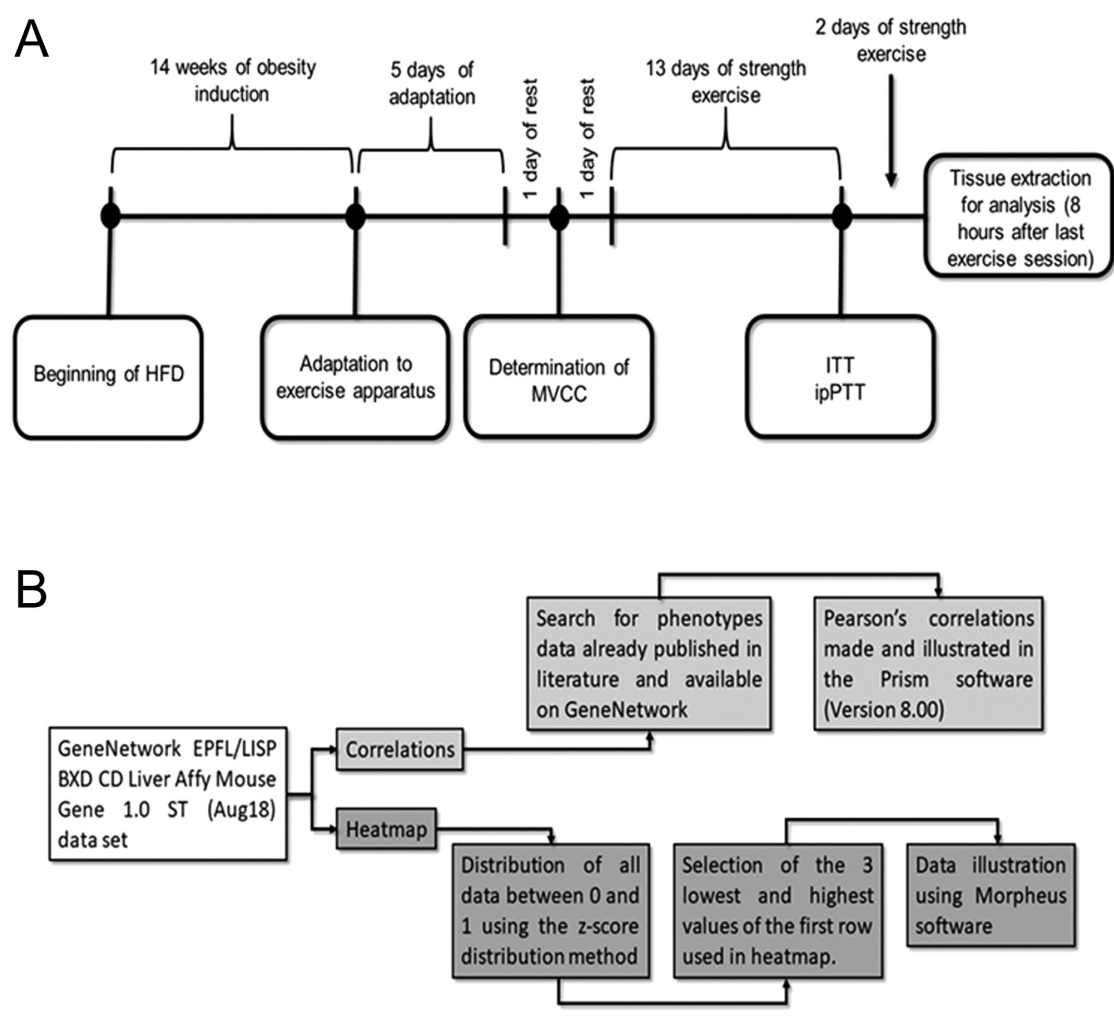

\begin{abstract}
Figure 1
Experimental design and pipeline schematic regarding bioinformatics analysis. (A) Schematic representation of the experiments. The insulin tolerance test (ITT), intraperitoneal pyruvate tolerance test (ipPTT), and tissue extraction were performed $8 \mathrm{~h}$ after the exercise session and $8 \mathrm{~h}$ of fasting. (B) Pipeline schematic describing the steps performed.
\end{abstract}

and after verification and assurance of the lack of corneal reflexes, the liver was collected and rapidly snap-frozen in liquid nitrogen and stored at $-80^{\circ} \mathrm{C}$ until analysis. The epididymal and retroperitoneal adipose tissue (right side) were removed and weighed to measure the fat depots. The liver was homogenized in extraction buffer $(1 \%$ Triton-X 100, $100 \mathrm{mM}$ Tris ( $\mathrm{pH} 7.4$ ), $100 \mathrm{mM}$ sodium pyrophosphate, $100 \mathrm{mM}$ sodium fluoride, $10 \mathrm{mM}$ EDTA, $10 \mathrm{mM}$ sodium vanadate, $2 \mathrm{mM}$ PMSF, and $0.1 \mathrm{mg}$ aprotinin $/ \mathrm{mL}$ ) at $4^{\circ} \mathrm{C}$ with a TissueLyser II (QUIAGEN ${ }^{\circledR}$ ) operated at maximum speed for $120 \mathrm{~s}$. The lysates were centrifuged (Eppendorf 5804R) at $12,851 \boldsymbol{g}$ at $4^{\circ} \mathrm{C}$ for 15 min to remove insoluble material, and the supernatant was used for the assay. The protein content was determined by the bicinchoninic acid method (Walker, 1994). The samples containing $60 \mu \mathrm{g}$ of total protein were applied to a polyacrylamide gel for separation by SDS-PAGE and transferred to nitrocellulose membranes. The membranes were blocked with 5\% dry milk at room temperature for $1 \mathrm{~h}$ and incubated with primary antibodies against the protein of interest. After that, a specific secondary antibody was used. The specific bands were labeled by chemiluminescence, and visualization was performed by the G:BOX photodocumentation system (Syngene). The bands were quantified using the
ImageJ 1.51s software. The primary antibodies used were: anti-Phospho-Akt ser473 (4060), anti-Akt (4685), and anti- $\beta$-actin (3700) from Cell Signaling Technology ${ }^{\circledR}$, and anti-PC (sc271493) from Santa Cruz Biotechnology ${ }^{\circledR}$. The secondary antibodies used were anti-rabbit and antimouse, from Cell Signaling Technology ${ }^{\circledR}$. More details about the antibodies used can be found in Supplementary Table 1 (see section on supplementary materials given at the end of this article).

\section{Reverse transcription and quantitative polymerase chain reaction ( $R T$ TPCR)}

Total RNA was extracted from the tissues using the TRIzol methodology(Invitrogen). ThecDNAwassynthesizedusing the High Capacity cDNA RT Kit (Thermo Fisher Scientific). The qPCR was performed using SYBR Green PCR Master Mix (Applied Biosystems). The following primers were evaluated: $P C$ - forward: GACGGCGAGGAGATAGTGTC; reverse: CATGGACTGTTCGGAACTTCA and Actb - forward: TGTCGAGTCGCGTCCA; reverse: TCATCCATGGCGAACTGGTG (used as the normalizing gene). Samples for this reaction, in triplicate, were made in a 96-well plate (MicroAmp, Applied Biosystems) for amplification and reading in the Step One Plus Real-Time 
PCR System (Applied Biosystems). The primers used were designed using the Primer-BLAST software (NCBI).

\section{Bioinformatics analysis}

All the data used in this bioinformatics analysis were provided by GeneNetwork (http://www.genenetwork. org/) using the EPFL/LISP BXD CD Liver Affy Mouse Gene 1.0 ST (Aug 18) data set. The Pearson's correlation was calculated using Prism (8.0.1) GraphPad Software with phenotypes provided by previous experiments available in GeneNetwork (Philip et al. 2010, Andreux et al. 2012, Williams et al. 2016) and heat maps were made using the Morpheus software (https://software.broadinstitute.org/ morpheus/). To better describe the bioinformatics analysis, a pipeline illustration was drawn and can be found in Fig. 1B. Briefly, the same data set was used to devise the correlations and the heat map. The $P C$ levels were correlated with phenotypes related to body composition, glucose metabolism, and locomotor activity. Moreover, to make the heat map, all data used were distributed in values between 0 and 1 using the z-score distribution method, and then the data were organized in ascending order and the three lowest and highest values of the first row (locomotor activity or fat mass) were used to create the heat map.

\section{Statistical analysis}

The results were shown as the mean \pm S.E.M.. The ShapiroWilk test was used to evaluate the Gaussian distribution of the data, and Student's $t$-test was used to compare the two groups with parametric data when necessary. Furthermore, to compare more than two groups, the one-way ANOVA test was performed, followed by Bonferroni's post hoc test. To analyze the points of the ITT and ipPTT, the twoway ANOVA (with repeated measures when appropriate) was used, with Bonferroni's correction for multiple comparisons. The statistical significance level considered was $P<0.05$. The graphics were created and the statistical analysis performed using Prism (7.00) GraphPad Software.

\section{Results}

\section{Short-term strength training reduces blood glucose levels and increases insulin sensitivity in obese mice, even without changes in body adiposity}

After obesity induction with a HFD, the animals in the ObS group presented increased body mass, fasting glycemic levels, and body adiposity compared to the Ctl animals (Fig. 2A, B, C and D). However, after 15 strength training
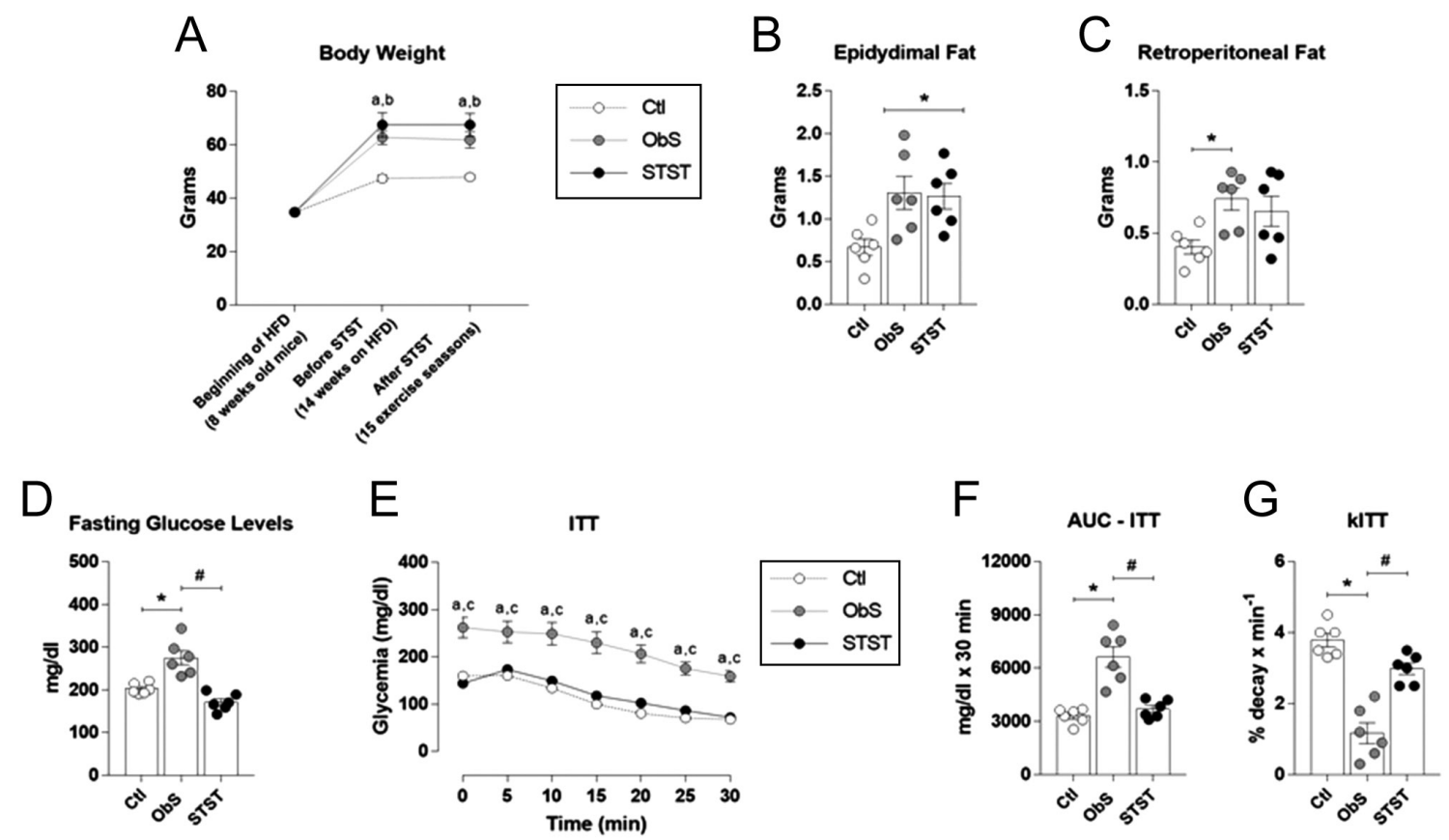

Figure 2

Physiological parameters of experimental groups. (A) Body weight at beginning of HFD, before and after STST protocol. (B and C) Weight of epididymal and retroperitoneal fat, respectively. (D) Fasting glucose levels after $8 \mathrm{~h}$ of fasting. (E) Glucose levels during ITT. (F) Area under the curve during ITT. (G) Rate constant for plasma glucose disappearance. In A and E: a $P<0.05$ for Ctl vs ObS; ${ }^{b} P<0.05$ for CtI vs STST; $c P<0.05$ for ObS vs STST. In B, C, D, F, and G: $* P<0.05$ vs $\mathrm{Ctl} ; \# P<0.05$ vs ObS ( $n=6$ per group). 
sessions, the animals in the STST group showed identical fasting glycemic levels to the $\mathrm{Ctl}$ group, even without changes in body composition (Fig. 2A, B, C and D). After insulin injection, the animals in the ObS group showed higher glycemic levels and impaired kITT compared to the $\mathrm{Ctl}$ group, reinforcing the insulin resistance status in these animals (Fig. 2E, F and G). But the STST group showed the same pattern as the lean animals, with no difference in the AUC and kITT (Fig. 2E, F and G). More details about Fig. 2 can be found in Supplementary Figure 2 .

\section{Obese trained animals showed increased hepatic insulin sensitivity and better control of HGP compared to sedentary obese animals}

In the next step, the effects of obesity and short-term strength training were checked using parameters related to hepatic insulin sensitivity and control of HGP. Eight hours after the 13th strength training session, the animals from the three experimental groups were subjected to the ipPTT, receiving an intraperitoneal injection of pyruvate solution and having their glycemic values checked every
$30 \mathrm{~min}$, from 0 to $120 \mathrm{~min}$ into the experiment. As shown in Fig. 3A, the animals in the ObS group showed increased glycemic values compared to the animals in the Ctl group throughout the $120 \mathrm{~min}$ of the test. However, the animals in the STST group showed a reduction in these values at all points. Consequently, the AUC during the test was higher for the ObS group compared to the other two groups, while the STST group showed no difference from the Ctl group (Fig. 3B).

To assess the hepatic insulin sensitivity, we measured hepatic pAktser473 levels after an intraperitoneal insulin injection, since Akt activation is a critical step of the insulin-signaling pathway in the liver (Kubota et al. 2017). We observed that the ObS group animals had significant hepatic insulin resistance, with reduced levels of pAkt ${ }^{\text {ser } 473}$ even without changes in total Akt levels (Fig. 3C, D and E). On the other hand, the trained animals presented increased Akt levels and phosphorylation compared to the animals in the ObS group (Fig. 3F, G and $\mathrm{H}$ ), showing that STST is a new strategy to increase hepatic insulin sensitivity in obese animals. More details about Fig. 3 can be found in Supplementary Figure 3.
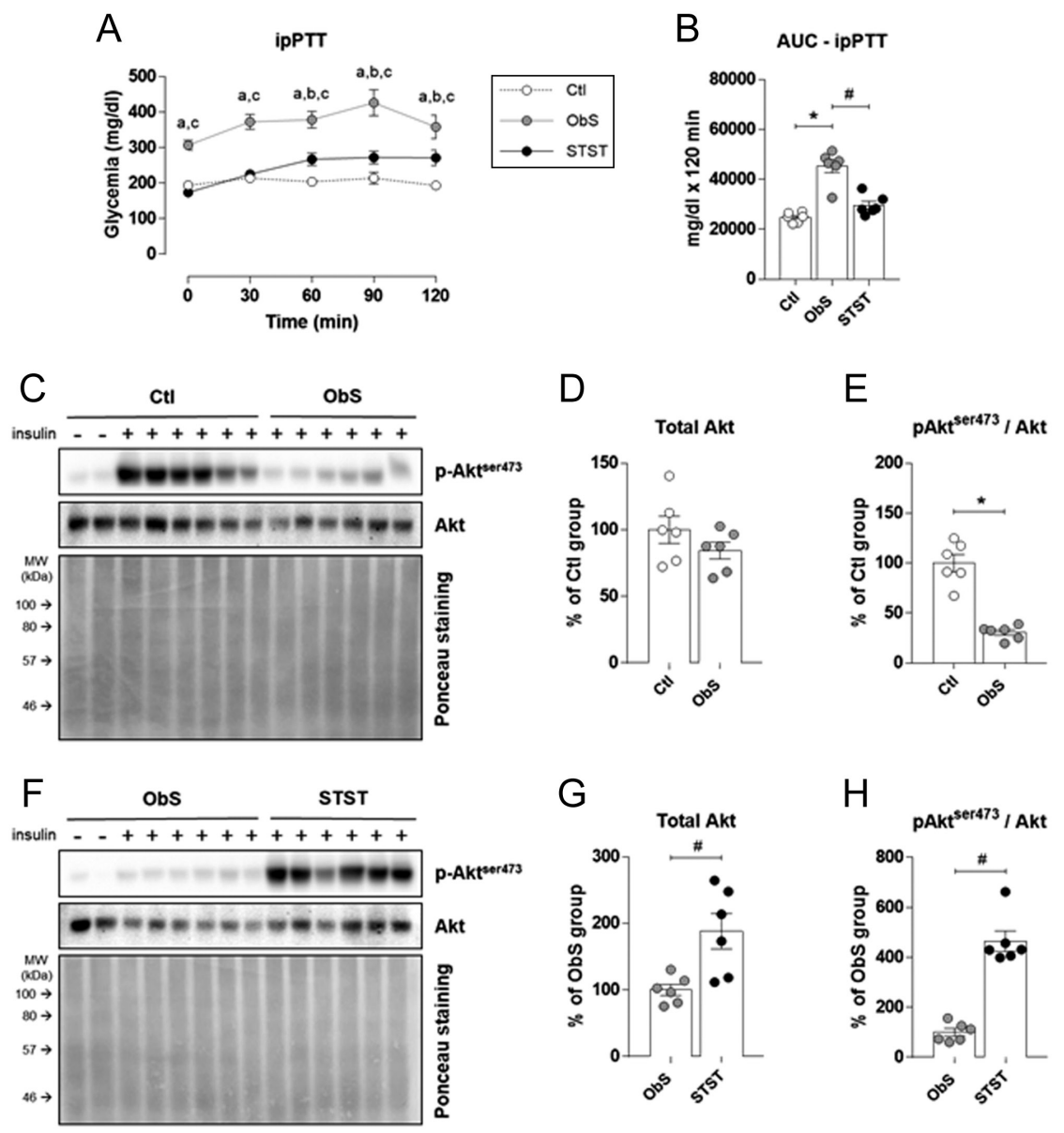

Figure 3

ipPTT and hepatic insulin sensitivity. (A) Glucose levels during ipPTT. (B) Area under the curve during ipPTT. (C) Bands of hepatic pAktser473 and total Akt of $\mathrm{Ctl}$ and ObS groups after insulin injection. (D) Quantification of total Akt levels of $\mathrm{Ctl}$ and ObS groups. (E) Quantification of hepatic pAktser473/Akt of $\mathrm{Ctl}$ and ObS groups. (F) Bands of hepatic pAktser473 and total Akt of ObS and STST groups after insulin injection. (G) Quantification of total Akt levels of ObS and STST groups. (H) Quantification of hepatic pAktser473/Akt of ObS and STST groups. Only the bands of the animals stimulated with insulin were quantified. The quantification of total Akt levels were normalized by Ponceau staining, and pAkt $\mathrm{ser}^{\mathrm{s} 43}$ was normalized by total Akt quantification. In A: a $P<0.05$ for Ctl vs ObS; b $P<0.05$ for CtI vs STST; $c P<0.05$ for ObS vs STST. In B, D, E, G, and H: $\star P<0.05$ vs Ctl; $\# P<0.05$ vs ObS ( $n=6$ per group). https://joe.bioscientifica.com https://doi.org/10.1530/JOE-20-0193
(C) 2020 Society for Endocrinology Published by Bioscientifica Ltd. Printed in Great Britain 
A

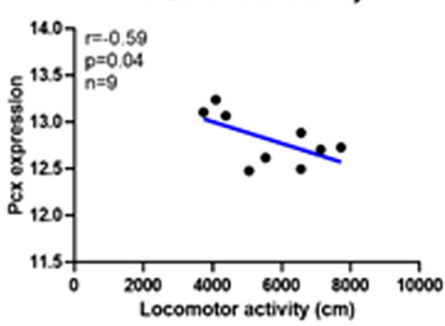

D
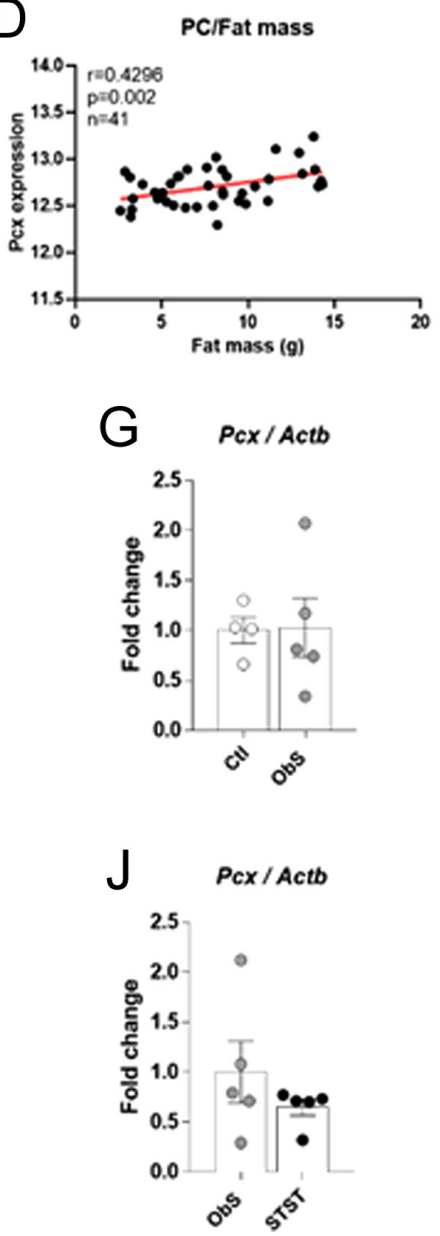

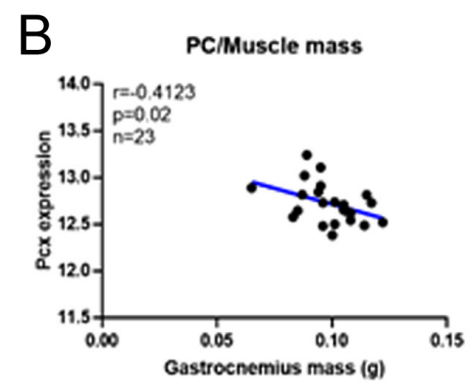

E

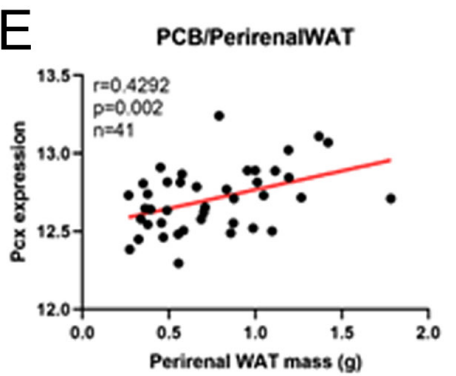

$\mathrm{H}$

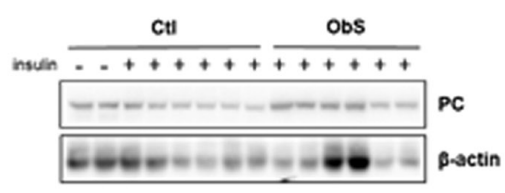

K

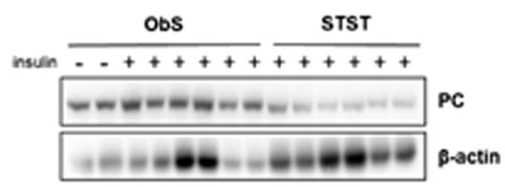

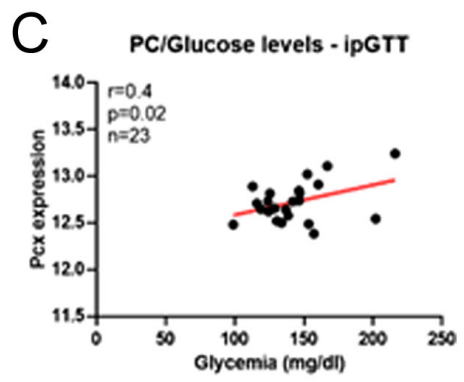

F
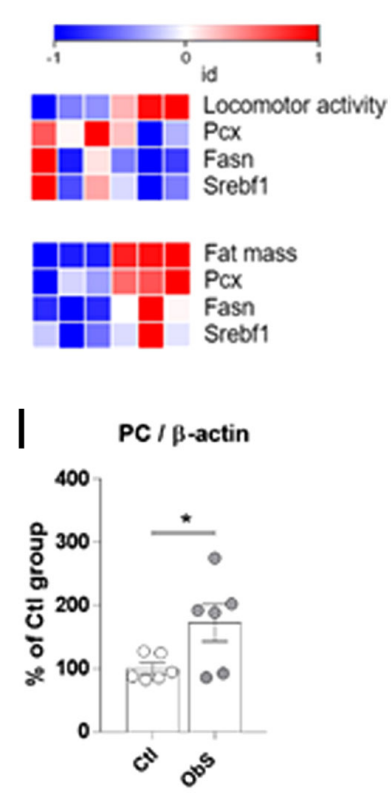

L

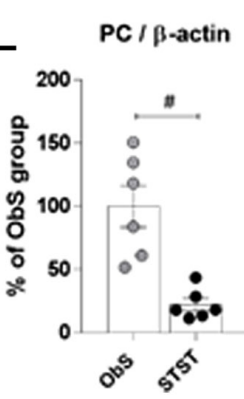

Figure 4

Bioinformatics analyses and hepatic PC levels. (A) locomotor activity; (B) muscle mass; (C) blood glucose levels in GTT 60 min after glucose intraperitoneal injection; (D) fat mass; (E) perirenal WAT mass. Blue lines represent negative correlations and red lines represent positive correlations. (F) Heat map showing how the lipogenic genes decrease with more activity stimulus and increase with fat accumulation. (G) Hepatic PC/Actb mRNA levels of Ctl and ObS groups ( $n=4-5$ per group). (H) Western blot analysis of hepatic PC and $\beta$-actin of Ctl and ObS groups ( $n=6$ per group). (I) Quantification of bands of hepatic $\mathrm{PC} / \beta$-actin of CtI and ObS groups. () Hepatic PC/Actb mRNA levels of ObS and STST groups ( $n=4-5$ per group). Only the bands of the animals stimulated with insulin were quantified. (K) Western blot analysis of hepatic PC and $\beta$-actin of ObS and STST groups ( $n=6$ per group). (L) Quantification of bands of hepatic PC/ $\beta$-actin of ObS and STST groups. * $P<0.05$ vs Ctl; $\# P<0.05$ vs ObS. A full color version of this figure is available at https://doi.org/10.1530/JOE-20-0193.

\section{Bioinformatics analysis and effects of obesity and short-term strength training on hepatic PC levels}

To check the relevance of PC in overall metabolic control, we first evaluated the correlation between hepatic $P C$ levels and several phenotypes using a public database with a large panel of isogenic BXD mice strains. We found that the expression of this $P C$ gene was positively correlated with glucose levels, fat mass, and peritoneal WAT, revealing the harmful effects of obesity on PC levels 
(Fig. $4 \mathrm{~A}, \mathrm{~B}, \mathrm{C}, \mathrm{D}$ and $\mathrm{E}$ ). On the other hand, the $P C$ expression was negatively correlated with locomotor activity and muscle mass, indicating that physical activity might be an alternative to reduce PC levels. Moreover, the first heat map (Fig. 4F) shows that with an increase in locomotor activity, both $P C$ expression and lipogenic genes (Fasn and Srebpf1) decrease. In contrast, the opposite effect is shown in the second heat map, where the expression of those genes increases with the highest fat mass levels.

Finally, we investigated whether obesity and STST can change PC levels in mice hepatocytes. Initially, we observed that obesity increased the hepatic PC protein levels of obese mice (Fig. $4 \mathrm{H}$ and I), even without changing the mRNA levels of PC (Fig. 4G). In contrast, after 15 sessions of strength training, the trained animals presented reduced PC protein levels (Fig. $4 \mathrm{~K}$ and L), also without changes in $P C$ mRNA levels (Fig. 4J). More details about Fig. 4 can be found in Supplementary Figure 4 .

\section{Discussion}

Insulin resistance is indicated as the main factor responsible for the onset of T2DM and cardiovascular diseases related to hyperglycemia (Patel \& Goyal 2019). By 2050 , it is estimated that $33 \%$ of the US population will be diagnosed with insulin resistance, and it will be one of the main causes of death (Boyle et al. 2010). In this context, hepatic insulin resistance plays a fundamental role in hepatic glucose production (HGP), since it is known that the failure of insulin to inhibit HGP is a crucial factor in the perpetuation of hyperglycemia in diabetic patients (Magnusson et al. 1992, Petersen \& Shulman 2017). Several studies demonstrate that obesity is strongly associated with hepatic insulin resistance, and despite the significant advances of medicine (Tilg et al. 2017, Foretz et al. 2019), lifestyle changes such as physical training, leading to increased energy expenditure and reduced body adiposity, are still the most promising interventions with the best results (Bacchi et al. 2013, Sargeant et al. 2018, Pereira et al. 2019). However, the biomolecular mechanisms involved in the increased hepatic insulin sensitivity in obese patients provided by physical exercise remains poorly understood. In the present study, we demonstrated that a short period of strength training was able to increase insulin sensitivity and hepatic Akt phosphorylation in response to insulin in obese mice, culminating in the improved control of HGP and normalization of fasting blood glucose levels.
Furthermore, we also showed that the training reversed the increment in hepatic PC content induced by obesity, indicating a new mechanism by which strength training can be classified as an essential strategy against hepatic insulin resistance. Another important point of our study is that we did not observe any reduction in fat deposits after the exercise protocol, showing that the aforementioned effects were directly caused by the training, and were not side effects related to the decrease in body adiposity after the long-term exercise protocol.

Previously to short-term strength training, the animals were subjected to DIO. We observed that the sedentary obese animals showed an increase in both body mass and body adiposity, providing an increase in fasting glucose. However, after 15 sessions of strength training, the glycemic values of the trained animals returned to normal levels, showing no difference from the lean animals, thus corroborating data previously found both in rodents subjected to the same conditions (Pereira et al. 2019) and in women with liver insulin resistance who performed strength training for 4 months (Honka et al. 2016). We also observed that the animals in the ObS group showed reduced insulin sensitivity, with higher glycemic levels and less glucose uptake after intraperitoneal insulin injection, as is well described in the literature (Oliveira et al. 2015, Roden \& Shulman 2019). Previous studies have already shown that strength training can be an important tool against obesity-associated insulin resistance (Klimcakova et al. 2006, Tang et al. 2014). After 8 weeks of strength training, obese rats showed both an improvement in insulin action and higher glucose tolerance, assessed by the ipITT and oral glucose tolerance test, respectively (Tang et al. 2014). Similar, data were found in a study involving obese male subjects that showed increased insulin sensitivity in the euglycemic-hyperinsulinemic clamp after 3 months of dynamic strength training (Klimcakova et al. 2006). In the present study, we showed that a short period of strength training is also able to increase insulin action in obese mice, providing higher glucose uptake and reversing hyperglycemia.

Similarly, the trained animals also showed more activation of Akt and better control of HGP compared to the animals in the ObS group, reversing obesity-induced liver insulin resistance. There are already a large number of studies showing that aerobic training provides several benefits to the liver in obesity conditions (Pereira et al. 2017, Sargeant et al. 2018). After 8 weeks of swimming, obese mice showed higher phosphorylation of hepatic Akt after insulin injection, also with reduced fasting glycemia and independently of any adiposity reduction 
et al. 2013). Similarly, our bioinformatics analysis, which

(Marinho et al. 2012). The authors also observed improved control of HGP in the trained animals, with lower glycemic values at all points in the ipPTT (Marinho et al. 2012). A recent meta-analysis involving 94 participants from six different studies showed that aerobic training can increase insulin action in the liver of overweight subjects (Sargeant et al. 2018). Another important finding was that strength training also provided an increase in total Akt levels. It is widely demonstrated that despite the lower activation of Akt in the liver of obese animals with non-alcoholic fatty liver disease (NAFLD), their total protein levels remain unchanged (Xiao et al. 2018, Xu et al. 2020). Similarly, several studies using obese rodents subjected to aerobic exercise have not observed changes in Akt levels, not even after an acute session (Ropelle et al. 2009) such as long-term aerobic training (Oliveira et al. 2011, Muñoz et al. 2018). However, in the present study, the animals subjected to the short-term strength training protocol showed an increase in total hepatic Akt content, revealing another mechanism by which strength training contributes to an increase in insulin action in NAFLD. However, despite the initial evidence showing that strength training provides significant metabolic improvements in obesity conditions (Klimcakova et al. 2006, Pereira et al. 2017), the mechanisms related to these benefits remain poorly explored.

Recently, our research group demonstrated that after 15 sessions of strength training, obese mice showed reduced hepatic lipogenesis, fatty acid synthase (FAS), and acetyl-CoA carboxylase (ACC) contents, and increased ACC phosphorylation, suppressing their lipogenic activity (Pereira et al. 2019). Thus, the hepatic lipid content was reduced, increasing insulin action and the control of HGP. However, the mechanisms that control hepatic gluconeogenesis in these animals have not been explored. We suggest that short-term strength training can provide better control of HGP, reducing hepatic PC content. As previously discussed, PC is known to be an essential protein in HGP control, mediating the conversion of pyruvate to OXA and initiating gluconeogenesis (Utter \& Keech 1960). Kumashiro and colleagues observed that HFD-induced obesity increased hepatic PC content, although their transcripts did not change (Kumashiro et al. 2013). However, the use of ASO to reduce PC expression concomitant with a HFD provided better control of HGP in rodents, since they showed reduced glycemic values at all points during the ipPPTT, increased Akt phosphorylation, and lower basal endogenous glucose production, confirming the relevant participation of PC in maintaining healthy blood glucose levels (Kumashiro includes a large panel of isogenic BXD mice strains, revealed that mice with higher hepatic $P C$ transcription had elevated glycemic values during a glucose tolerance test, thus corroborating our data showing increased PC levels and impaired insulin sensitivity in sedentary obese mice. Furthermore, our bioinformatics analysis also demonstrated a strong negative correlation between $P C$ levels and both locomotor activity and muscle mass, two phenotypes commonly associated with physical exercise (Ross et al. 2019), thus reinforcing our hypothesis that the metabolic benefits provided by strength training could be mediated by mechanisms involving PC.

Finally, we assessed whether strength training was able to alter the protein content of hepatic PC. Muñoz and colleagues demonstrated that aerobic exercise can lead to an improvement in the hepatic metabolism of obese rodents by reducing PC levels (Muñoz et al. 2018). However, the effects of strength training in this context and without changes in body adipose remain unexplored. In the mitochondria, when there is an excess of energy, high levels of Acetyl-CoA activate PC and stimulate the conversion of pyruvate to OXA to initiate the formation of a new glucose molecules. When there are high levels of mitochondrial $\mathrm{NADH}$, the mitochondrial malate dehydrogenase converts OXA to malate so it can be carried to the cytoplasm. Then, in the reduction process $\left(\mathrm{NAD}^{+}\right.$to $\mathrm{NADH})$, cytosolic malate dehydrogenase converts malate into OXA again (Jitrapakdee et al. 2008). Furthermore, this cytoplasmic OXA is converted to phosphoenolpyruvate by PEPCK and, after passing through a reverse glycolysis process, the G6Pase enzyme is able to remove a phosphate group from the glucose-6-phosphate molecule, facilitating its access to the circulatory system (Weber \& Cantero 1954). In the present study, it was possible to observe that only 15 strength exercise sessions were sufficient to reduce the HGP of obese animals. According to the data obtained in this study, we suggest that this improvement in the animals' fasting hyperglycemia was mediated by the reduction in PC levels. Because the exercised obese animals showed a reduction in PC content, consequently, it is estimated that there was a reduction in the intracellular OXA levels and, then, lower substrate availability for gluconeogenesis. Nevertheless, these animals showed an improvement in hepatic insulin sensitivity, which may have favored the attenuation of PEPCK and G6Pase synthesis, thus contributing to a reduction in gluconeogenesis.

Therefore, we provide fresh evidence about a new mechanism by which strength training acts against 


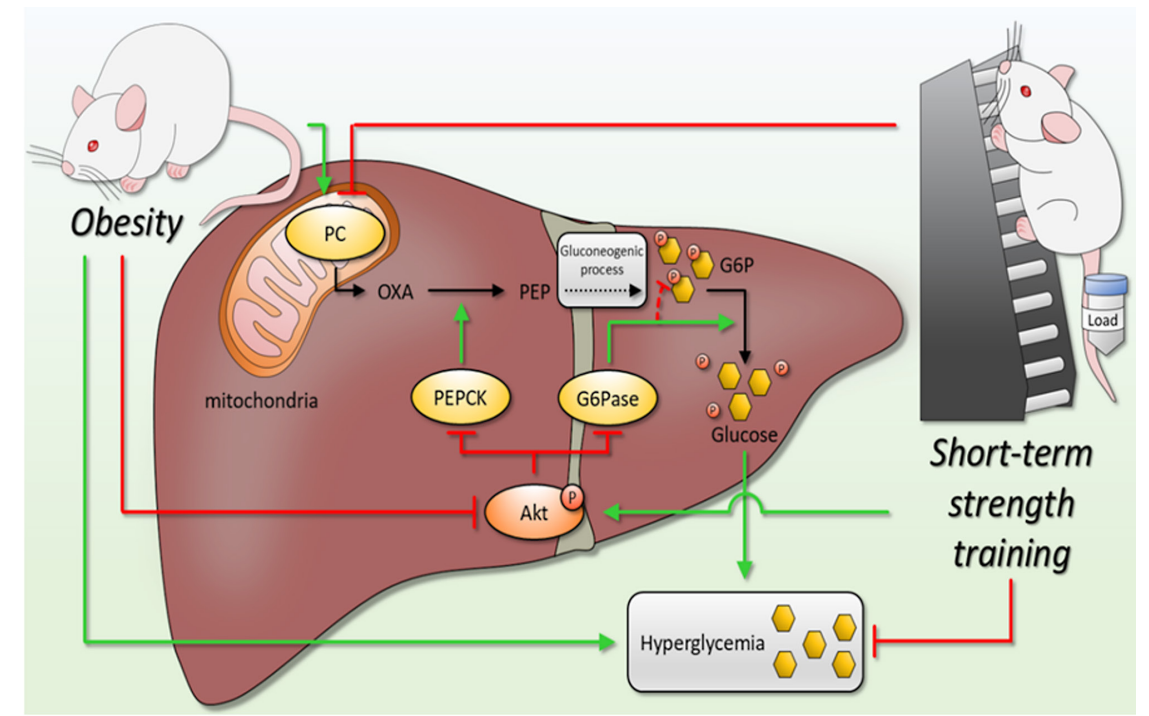

\section{Figure 5}

Schematic mechanism of the effect of short-term strength training on hepatic PC levels, independent of body weight changes. Obesity increases hepatic PC levels and reduces hepatic insulin action and HGP control, causing hyperglycemia. Short-term strength training reduces hepatic $P C$ levels and counteracts these adverse metabolic effects in obese mice, even without changes in adiposity. This is a new biomolecular mechanism by which short-term strength training is an efficient tool against obesity-related hyperglycemia and T2DM. A full color version of this figure is available at https:// doi.org/10.1530/JOE-20-0193. metabolic complications. As already aforementioned, previous studies have shown that strength training can reduce the protein content and the activity of lipogenic proteins in obese mice (Pereira et al. 2019), corroborating previous data observed in humans (Bacchi et al. 2013), obese rats (dos Santos et al. 2019), and ovariectomized rats (Domingos et al. 2012) after long-term strength training. With the reduction in liver fat, the insulin action and the control of HPG in these animals were improved (Pereira et al. 2019); however, the relationship between NAFLD and liver insulin resistance remains poorly understood. As shown in the heat map in Fig. 4F, mice with reduced levels of PC mRNA also showed a reduction in the expression of lipogenic genes (Fasn and Srebf1). Therefore, these data allow us to conclude that changes in the hepatic content of PC mediated by obesity may be related to the control of both HGP and fat liver accumulation. On the other hand, even though our bioinformatics analysis showed a positive correlation between hepatic levels of $P C$ mRNA and both total fat mass and perineal white adipose tissue, the STST animals showed reduced PC protein content without reducing adiposity, indicating that strength exercise might change PC metabolism through a non-adiposity related mechanism. Interestingly, we found no changes in $P C$ mRNA levels in the ObS and STST groups, confirming previous data found in human livers (Kumashiro et al. 2013) and suggesting that changes in liver PC protein levels caused by obesity and strength training are related to post-transcriptional changes. However, more studies are needed to understand these mechanisms better.

In conclusion, short-term strength training reduced hepatic PC levels in obese mice, increasing insulin action in the liver, and counteracting HFD-induced insulin resistance and hyperglycemia, as summarized in Fig. 5. Therefore, we suggest a new mechanism by which shortterm strength training can be an efficient tool against hepatic metabolic disorders associated with obesity. Importantly, all these results were observed independently of a reduction in body adiposity.

\section{Supplementary materials}

This is linked to the online version of the paper at https://doi.org/10.1530/ JOE-20-0193.

\section{Declaration of interest}

The authors declare that there is no conflict of interest that could be perceived as prejudicing the impartiality of the research reported.

\section{Funding}

The present work received financial support from the São Paulo Research Foundation (FAPESP; case numbers 2016/12569-6, 2016/24406-4, and 2015/07199-2).

\section{Author contribution statement}

L P M designed the paper. R M P and K C C R wrote the paper and had the overall responsibilities for the experiments and the truth of the results in this study. R M P, K C C R, M R S, G F P, and A P M performed the experiments and data collection. $R M P$ performed the statistical analysis. A P M performed the RTqPCR analysis. G F P performed the bioinformatics analysis. L P M contributed to the financial support. F M S, A S R S, D E C, $E R R, J R P$, and LPM contributed to the discussion and provided laboratory support. All the authors have read and approved this manuscript.
(C) 2020 Society for Endocrinology Published by Bioscientifica Ltd. Printed in Great Britain 


\section{Acknowledgements}

The authors would like to thank FAPESP (2015/07199-2, 2016/12569-6, 2016/24406-4), CNPq, FAEPEX, and CAPES for their financial support.

\section{References}

Andreux PA, Williams EG, Koutnikova H, Houtkooper RH, Champy MF, Henry H, Schoonjans K, Williams RW \& Auwerx J 2012 Systems genetics of metabolism: the use of the BXD murine reference panel for multiscalar integration of traits. Cell 150 1287-1299. (https://doi. org/10.1016/j.cell.2012.08.012)

Bacchi E, Negri C, Targher G, Faccioli N, Lanza M, Zoppini G, Zanolin E, Schena F, Bonora E \& Moghetti P 2013 Both resistance training and aerobic training reduce hepatic fat content in type 2 diabetic subjects with nonalcoholic fatty liver disease (the RAED2 Randomized Trial). Hepatology 58 1287-1295. (https://doi.org/10.1002/hep.26393)

Basu R, Chandramouli V, Dicke B, Landau B \& Rizza R 2005 Obesity and type 2 diabetes impair insulin-induced suppression of glycogenolysis as well as gluconeogenesis. Diabetes 54 1942-1948. (https://doi. org/10.2337/diabetes.54.7.1942)

Bonora E, Moghetti P, Zancanaro C, Cigolini M, Querena M, Cacciatori V Corgnati A \& Muggeo M 1989 Estimates of in vivo insulin action in man: comparison of insulin tolerance tests with euglycemic and hyperglycemic glucose clamp studies. Journal of Clinical Endocrinology and Metabolism 68 374-378. (https://doi.org/10.1210/jcem-68-2-374)

Boyle JP, Thompson TJ, Gregg EW, Barker LE \& Williamson DF 2010 Projection of the year 2050 burden of diabetes in the US adult population: dynamic modeling of incidence, mortality, and prediabetes prevalence. Population Health Metrics 8 29. (https://doi. org/10.1186/1478-7954-8-29)

Domingos MM, Rodrigues MFC, Stotzer US, Bertucci DR, Souza MVC, Marine DA, Gatto Cdo V, de Araújo HSS \& de Andrade Perez SE 2012 Resistance training restores the gene expression of molecules related to fat oxidation and lipogenesis in the liver of ovariectomized rats. European Journal of Applied Physiology 112 1437-1444. (https://doi. org/10.1007/s00421-011-2098-6)

dos Santos GF, Veras ASC, de Freitas MC, McCabe J, Seraphim PM \& Teixeira GR 2019 Strength training reduces lipid accumulation in liver of obese Wistar rats. Life Sciences 235 116834. (https://doi. org/10.1016/j.lfs.2019.116834)

Foretz M, Guigas B \& Viollet B 2019 Understanding the glucoregulatory mechanisms of metformin in type 2 diabetes mellitus. Nature Reviews: Endocrinology 15 569-589. (https://doi.org/10.1038/s41574019-0242-2)

Gastaldelli A, Baldi S, Pettiti M, Toschi E, Camastra S, Natali A, Landau BR \& Ferrannini E 2000 Influence of obesity and type 2 diabetes on gluconeogenesis and glucose output in humans: a quantitative study. Diabetes 49 1367-1373. (https://doi.org/10.2337/diabetes.49.8.1367)

Honka MJ, Bucci M, Andersson J, Huovinen V, Guzzardi MA, Sandboge S, Savisto N, Salonen MK, Badeau RM, Parkkola R et al. 2016 Resistance training enhances insulin suppression of endogenous glucose production in elderly women. Journal of Applied Physiology 120 633-639. (https://doi.org/10.1152/japplphysiol.00950.2015)

Jitrapakdee S, St Maurice M, Rayment I, Cleland WW, Wallace JC \& Attwood PV 2008 Structure, mechanism and regulation of pyruvate carboxylase. Biochemical Journal 413 369-387. (https://doi. org/10.1042/BJ20080709)

Klimcakova E, Polak J, Moro C, Hejnova J, Majercik M, Viguerie N, Berlan M, Langin D \& Stich V 2006 Dynamic strength training improves insulin sensitivity without altering plasma levels and gene expression of adipokines in subcutaneous adipose tissue in obese men. Journal of Clinical Endocrinology and Metabolism 91 5107-5112. (https://doi.org/10.1210/jc.2006-0382)
Kubota T, Kubota N \& Kadowaki T 2017 Imbalanced insulin actions in obesity and type 2 diabetes: key mouse models of insulin signaling pathway. Cell Metabolism 25 797-810. (https://doi.org/10.1016/j. cmet.2017.03.004)

Kumashiro N, Beddow SA, Vatner DF, Majumdar SK, Cantley JL, GuebreEgziabher F, Fat I, Guigni B, Jurczak MJ, Birkenfeld AL et al. 2013 Targeting pyruvate carboxylase reduces gluconeogenesis and adiposity and improves insulin resistance. Diabetes 62 2183-2194. (https://doi. org $/ 10.2337 / \mathrm{db} 12-1311)$

Magnusson I, Rothman DL, Katz LD, Shulman RG \& Shulman GI 1992 Increased rate of gluconeogenesis in type II diabetes mellitus. A 13C nuclear magnetic resonance study. Journal of Clinical Investigation 90 1323-1327. (https://doi.org/10.1172/JCI115997)

Marinho R, Ropelle ER, Cintra DE, De Souza CT, Da Silva ASR, Bertoli FC, Colantonio E, D'Almeida V \& Pauli JR 2012 Endurance exercise training increases APPL1 expression and improves insulin signaling in the hepatic tissue of diet-induced obese mice, independently of weight loss. Journal of Cellular Physiology 227 2917-2926. (https://doi. org/10.1002/jcp.23037)

Matthews JN, Altman DG, Campbell MJ \& Royston P 1990 Analysis of serial measurements in medical research. BMJ 300 230-235. (https:// doi.org/10.1136/bmj.300.6719.230)

McGilvery RW \& Mokrasch LC 1956 Purification and properties of fructose-1, 6-diphosphatase. Journal of Biological Chemistry 221 909-917.

Muñoz VR, Gaspar RC, Crisol BM, Formigari GP, Sant'Ana MR, Botezelli JD, Gaspar RS, da Silva ASR, Cintra DE, de Moura LP et al. 2018 Physical exercise reduces pyruvate carboxylase (PCB) and contributes to hyperglycemia reduction in obese mice. Journal of Physiological Sciences 68 493-501. (https://doi.org/10.1007/s12576017-0559-3)

Oliveira AG, Carvalho BM, Tobar N, Ropelle ER, Pauli JR, Bagarolli RA, Guadagnini D, Carvalheira JB \& Saad MJ 2011 Physical exercise reduces circulating lipopolysaccharide and TLR4 activation and improves insulin signaling in tissues of DIO rats. Diabetes 60 784-796. (https://doi.org/10.2337/db09-1907)

Oliveira V, Marinho R, Vitorino D, Santos GA, Moraes JC, Dragano N, Sartori-Cintra A, Pereira L, Catharino RR, da Silva ASR et al. 2015 Diets containing $\alpha$-linolenic $(\omega 3)$ or oleic $(\omega 9)$ fatty acids rescues obese mice from insulin resistance. Endocrinology 156 4033-4046. (https:// doi.org/10.1210/en.2014-1880)

Patel BM \& Goyal RK 2019 Liver and insulin resistance: new wine in old bottle!!! European Journal of Pharmacology 862 172657. (https://doi. org/10.1016/j.ejphar.2019.172657)

Pereira RM, Botezelli JD, da Cruz Rodrigues KC, Mekary RA, Cintra DE, Pauli JR, da Silva ASR, Ropelle ER \& de Moura LP 2017 Fructose consumption in the development of obesity and the effects of different protocols of physical exercise on the hepatic metabolism. Nutrients 9 405. (https://doi.org/10.3390/nu9040405)

Pereira RM, Rodrigues KCDC, Anaruma CP, Sant'Ana MR, de Campos TDP, Gaspar RS, Canciglieri RDS, de Melo DG, Mekary RA, da Silva ASR et al. 2019 Short-term strength training reduces gluconeogenesis and NAFLD in obese mice. Journal of Endocrinology 241 59-70. (https://doi.org/10.1530/JOE-18-0567)

Perry RJ, Camporez J-PG, Kursawe R, Titchenell PM, Zhang D, Perry CJ, Jurczak MJ, Abudukadier A, Han MS, Zhang XM et al. 2015 Hepatic acetyl CoA links adipose tissue inflammation to hepatic insulin resistance and type 2 diabetes. Cell 160 745-758. (https://doi. org/10.1016/j.cell.2015.01.012)

Petersen MC \& Shulman GI 2017 Roles of diacylglycerols and ceramides in hepatic insulin resistance. Trends in Pharmacological Sciences 38 649-665. (https://doi.org/10.1016/j.tips.2017.04.004)

Philip VM, Duvvuru S, Gomero B, Ansah TA, Blaha CD, Cook MN, Hamre KM, Lariviere WR, Matthews DB, Mittleman G et al. 2010 High-throughput behavioral phenotyping in the expanded panel https://joe.bioscientifica.com https://doi.org/10.1530/JOE-20-0193
(C) 2020 Society for Endocrinology Published by Bioscientifica Ltd. Printed in Great Britain 
of BXD recombinant inbred strains. Genes, Brain, and Behavior 9 129-159. (https://doi.org/10.1111/j.1601-183X.2009.00540.x)

Reeves PG, Nielsen FH \& Fahey GC 1993 AIN-93 purified diets for laboratory rodents: final report of the American Institute of Nutrition ad hoc writing committee on the reformulation of the AIN-76A rodent diet. Journal of Nutrition 123 1939-1951. (https://doi. org/10.1093/jn/123.11.1939)

Roden M \& Shulman GI 2019 The integrative biology of type 2 diabetes. Nature 576 51-60. (https://doi.org/10.1038/s41586-019-1797-8)

Ropelle ER, Pauli JR, Cintra DE, Frederico MJS, De Pinho RA, Velloso LA \& De Souza CT 2009 Acute exercise modulates the FoxO1/PGC-1 $\alpha$ pathway in the liver of diet-induced obesity rats. Journal of Physiology 587 2069-2076. (https://doi.org/10.1113/ jphysiol.2008.164202)

Ross JM, Coppotelli G, Branca RM, Kim KM, Lehtiö J, Sinclair DA \& Olson L 2019 Voluntary exercise normalizes the proteomic landscape in muscle and brain and improves the phenotype of progeroid mice. Aging Cell 18 e13029. (https://doi.org/10.1111/ acel.13029)

Samuel VT, Beddow SA, Iwasaki T, Zhang XM, Chu X, Still CD, Gerhard GS \& Shulman GI 2009 Fasting hyperglycemia is not associated with increased expression of PEPCK or G6Pc in patients with type 2 diabetes. PNAS 106 12121-12126. (https://doi. org/10.1073/pnas.0812547106)

Sargeant JA, Gray LJ, Bodicoat DH, Willis SA, Stensel DJ, Nimmo MA, Aithal GP \& King JA 2018 The effect of exercise training on intrahepatic triglyceride and hepatic insulin sensitivity: a systematic review and meta-analysis. Obesity Reviews 19 1446-1459. (https://doi. org/10.1111/obr.12719)

Tang L, Luo K, Liu C, Wang X, Zhang D, Chi A, Zhang J \& Sun L 2014 Decrease in myostatin by ladder-climbing training is associated with insulin resistance in diet-induced obese rats. Chinese Medical Journal 127 2342-2349.
Tilg H, Moschen AR \& Roden M 2017 NAFLD and diabetes mellitus. Nature Reviews: Gastroenterology and Hepatology 14 32-42. (https://doi. org/10.1038/nrgastro.2016.147)

Utter MF \& Keech DB 1960 Formation of oxaloacetate from pyruvate and carbon dioxide. Journal of Biological Chemistry 235 PC17-PC18.

Walker JM 1994 The bicinchoninic acid (BCA) assay for protein quantitation. Methods in Molecular Biology 32 5-8. (https://doi. org/10.1385/0-89603-268-X:5)

Weber G \& Cantero A 1954 Glucose-6-phosphatase studies in fasting. Science 120 851-852. (https://doi.org/10.1126/science.120.3125.851)

Weinberg MB \& Utter MF 1980 Effect of streptozotocin-induced diabetes mellitus on the turnover of rat liver pyruvate carboxylase and pyruvate dehydrogenase. Biochemical Journal 188 601-608. (https:// doi.org/10.1042/bj1880601)

Williams EG, Wu Y, Jha P, Dubuis S, Blattmann P, Argmann CA, Houten SM, Amariuta T, Wolski W, Zamboni N et al. 2016 Systems proteomics of liver mitochondria function. Science $\mathbf{3 5 2}$ aad0189. (https://doi.org/10.1126/science.aad0189)

Xiao XH, Wang YD, Qi XY, Wang YY, Li JY, Li H, Zhang PY, Liao HL, Li MH, Liao ZZ et al. 2018 Zinc alpha2 glycoprotein protects against obesity-induced hepatic steatosis. International Journal of Obesity 42 1418-1430. (https://doi.org/10.1038/s41366-018-0151-9)

Xu M, Ge C, Zhu L, Qin Y, Du C, Lou D, Li Q, Hu L, Sun Y, Dai X et al. 2020 iRhom 2 promotes hepatic steatosis by activating MAP3K7-dependent pathway. Hepatology [epub]. (https://doi. org/10.1002/hep.31436)

Yoon JC, Puigserver P, Chen G, Donovan J, Wu Z, Rhee J, Adelmant G, Stafford J, Kahn CR, Granner DK et al. 2001 Control of hepatic gluconeogenesis through the transcriptional coactivator PGC-1. Nature 413 131-138. (https://doi.org/10.1038/35093050)

Zhang X, Yang S, Chen J \& Su Z 2018 Unraveling the regulation of hepatic gluconeogenesis. Frontiers in Endocrinology 9 802. (https://doi. org/10.3389/fendo.2018.00802)

Received in final form 31 July 2020

Accepted 14 August 2020

Accepted Manuscript published online 17 August 2020 (c) 2020 Society for Endocrinology Published by Bioscientifica Ltd.
Printed in Great Britain 